\title{
Level crossings of eigenvalues of the Schrödinger Hamiltonian of the isotropic harmonic oscillator perturbed by a central point interaction in different dimensions
}

\author{
S. Fassari ${ }^{1,2,3}$, M. Gadella ${ }^{1}$, M. L. Glasser ${ }^{1,4}$, L. M. Nieto ${ }^{1}$, F. Rinaldi ${ }^{2,3}$ \\ ${ }^{1}$ Departamento de Física Teórica, Atómica y Óptica, U. Valladolid, 47011 Valladolid, Spain \\ ${ }^{2}$ CERFIM, PO Box 1132, Via F. Rusca 1, CH-6601 Locarno, Switzerland \\ ${ }^{3}$ Dipartimento di Fisica Nucleare, Subnucleare e delle Radiazioni, \\ Univ. degli Studi Guglielmo Marconi, Via Plinio 44, I-00193 Rome, Italy \\ ${ }^{4}$ Department of Physics, Clarkson University, Potsdam, NY, 13699 USA
}

silvestro.fassari@uva.es, gadella@fta.uva.es, laryg@tds.net, luismiguel.nieto.calzada@uva.es,matfa62@gmail.com

PACS 02.30.Gp, 02.30.Lt, 02.30.Sa, 02.30.Tb, 03.65.Db, 03.65.Ge, 68.65.Hb

DOI 10.17586/2220-8054-2018-9-2-179-186

In this brief presentation, some striking differences between level crossings of eigenvalues in one dimension (harmonic or conic oscillator with a central nonlocal $\delta^{\prime}$-interaction) or three dimensions (isotropic harmonic oscillator with a three-dimensional delta located at the origin) and those occurring in the two-dimensional analogue of these models will be highlighted.

Keywords: harmonic oscillator, point interactions, renormalisation, eigenvalues, level crossing.

Received: 8 January 2018

Revised: 24 January 2018

\section{Introduction}

The aim of this note is to compare the discrete spectrum of the isotropic harmonic oscillator perturbed by a point interaction centered at the origin, the bottom of a parabolic well, across various dimensions. The physical motivation behind this detailed study is the fact that this Hamiltonian, at least in the three-dimensional case, has been used over the last twenty years to model two-dimensional quantum wires and three-dimensional quantum dots.

It is important to point out and is well known that, while in two or three dimensions there is only one type of point interaction, namely the 2/3D delta (requiring a renormalization procedure if it is to be a perturbation of the negative Laplacian or any other free Hamiltonian), in one-dimensional Quantum Mechanics we have four different types of point interactions, out of which, the only one requiring such renormalization is the nonlocal $\delta^{\prime}$-interaction, defined by its quadratic form $\left(\cdot,\left|\delta^{\prime}\right\rangle\left\langle\delta^{\prime}\right|, \cdot\right)$ (see [1-3]). A self-adjoint Hamiltonian with the nonlocal $\delta^{\prime}$-interaction can also be defined as the norm resolvent limit of a Hamiltonian involving three Dirac distributions (see [4-6]). We remind the reader that this interaction, being an element of $\mathcal{H}_{-2} \backslash \mathcal{H}_{-1}$, may not be combined with the Dirac distribution, obviously an element of $\mathcal{H}_{-1}$ (the reader is referred to [2] for a thorough description of the Sobolev spaces labelled by a negative index).

While the spectral features of the three-dimensional isotropic oscillator perturbed by a single point interaction centered at the origin have been extensively investigated (see [7-10]), less attention has been paid to those of its two-dimensional analog. The remarkable spectral feature of the three-dimensional case is the existence of level crossings: each eigenvalue created by the point perturbation, clearly affecting only the $s$-states of the model, regarded as a function of either the parameter $\alpha$ labelling the possible self-adjoint extensions or its reciprocal $\beta$ (acting as the coupling constant), crosses the next lower unperturbed energy level (pertaining to an antisymmetric state with an odd value of the total angular momentum). Even more remarkable, all the level crossings take place for the same coupling parameter value.

As was shown in [11] (respectively [12,13]), level crossings also occur in the entirely discrete spectrum of the one-dimensional harmonic (respectively conic) oscillator perturbed by a nonlocal $\delta^{\prime}$-interaction. This onedimensional model shares the property of having all the crossings situated at the same point when either the extension parameter or the coupling parameter is chosen. However, the nature of such crossings is different from the aforementioned in three dimensions, as the eigenenergy of each antisymmetric bound state, regarded as a function of either the extension parameter $\alpha$, labeling the self-adjoint extensions, or the coupling $\beta$, its reciprocal, crosses the next lower unperturbed energy level (pertaining to a symmetric state). With reference to the terminology 
introduced in [14], one could describe the one-dimensional model as Fermionic and the three-dimensional model as Bosonic.

Therefore, we believe it may be worth investigating the spectral properties of the two-dimensional analog in depth given the increasing importance of this particular dimension in modern Nanophysics. As expected, level crossings also occur in the two-dimensional case. However, it will be seen that the location of these crossings is no longer at a unique parameter value. Furthermore, while in odd dimensions the interaction must be attractive in order to produce the crossings, a weaker repulsive interaction will suffice in the two-dimensional model.

\section{Level crossings in the discrete spectrum of the one-dimensional harmonic or conic oscillator perturbed by a central nonlocal $\delta^{\prime}$-interaction}

In this section, we shall review the findings of [11] (respectively $[12,13]$ ) showing the occurrence of level crossings in the discrete spectrum of the harmonic (resp. conic) oscillator perturbed by a nonlocal $\delta^{\prime}$-interaction centred at the minimum of the parabolic (resp. V-shaped) potential.

It is well known that the Schrödinger Hamiltonian perturbed by a Dirac distribution need not be defined by means of renormalization, since such a perturbation is infinitesimally small in the sense of quadratic forms (see [1,2]). Therefore, the Dirac distribution can be regarded as an element of $\mathcal{H}_{-1}$.

As anticipated earlier, the nonlocal $\delta^{\prime}$-perturbation of the one-dimensional negative Laplacian, being an element of $\mathcal{H}_{-2} \backslash \mathcal{H}_{-1}$, requires instead either the self-adjoint extension method or coupling constant renormalisation to produce a self-adjoint Hamiltonian (see [1,2]).

The spectral effects of such a singular perturbation on the harmonic oscillator were studied in [11] starting from the explicit expression for the resolvent of the self-adjoint operator $H_{\beta}$ acting like $H_{0}=\frac{1}{2}\left[-\frac{d^{2}}{d x^{2}}+x^{2}\right]$ on the functions satisfying the condition $\psi\left(0^{+}\right)-\psi\left(0^{-}\right)=-\beta \psi^{\prime}(0)$ :

$$
\left[H_{\beta}-E\right]^{-1}=\left[H_{0}-E\right]^{-1}+\frac{2|\Psi(E)\rangle\langle\Psi(E)|}{\frac{1}{\beta}-\frac{1}{\beta_{0}}+\frac{2 \Gamma(3 / 4-E / 2)}{\Gamma(1 / 4-E / 2)}},
$$

with $\beta_{0}=\frac{\Gamma(1 / 4)}{2 \Gamma(3 / 4)} \approx 1.47934$ and:

$$
\Psi(x ; E)=\sum_{n=0}^{\infty} \frac{(2 n+1)^{1 / 2} \psi_{2 n}(0)}{(2 n+3 / 2-E)} \psi_{2 n+1}(x),
$$

$\psi_{n}(x)$ being the $n$-th normalized eigenfunction of the harmonic oscillator. The latter function, having a jump discontinuity satisfying the condition $\psi\left(0^{+}\right)-\psi\left(0^{-}\right)=-\beta \psi^{\prime}(0)$, is square summable since the sequence $\psi_{2 n}(0)$ behaves like $n^{-1 / 4}$ as $n \rightarrow \infty$. Hence, the equation determining the bound state energies of $H_{\beta}$ is:

$$
\frac{1}{\beta_{0}}-\frac{1}{\beta}=\frac{2 \Gamma(3 / 4-E / 2)}{\Gamma(1 / 4-E / 2)} .
$$

Clearly, given that the perturbation does not affect the symmetric subspace, the even eigenvalues $E_{2 n}=$ $2 n+1 / 2$ of the unperturbed harmonic oscillator are still in the discrete spectrum for any value of $\beta$. Each new odd energy level created by the perturbation is a decreasing function of the coupling parameter $\beta$ (as can be easily seen by means of implicit differentiation), namely $E_{2 n+1}(\beta)$. For $\beta=0$, the operator trivially coincides with $H_{0}$, so the odd eigenvalues are also those of the harmonic oscillator $E_{2 n+1}=2 n+3 / 2$. Something remarkable occurs for $\beta=\beta_{0}$ : as the left hand side of (3) vanishes, the only values of the energy that can satisfy (3) are those that make the Gamma function in the denominator diverge, which implies that $1 / 4-E / 2=-n$, or equivalently $E_{2 n+1}\left(\beta_{0}\right)=2 n+1 / 2$. Therefore, the discrete spectrum of $H_{\beta_{0}}$ consists only of the doubly degenerate eigenvalues $E_{2 n}\left(\beta_{0}\right)=E_{2 n+1}\left(\beta_{0}\right)=2 n+1 / 2$. As $E_{2 n+1}(\beta)<E_{2 n}(\beta)=2 n+1 / 2$ for any $\beta>\beta_{0}$, we have infinitely many level crossings to the right of the critical value $\beta_{0}$ of the coupling parameter. The plot shown in Fig. 1 depicts the three lowest level crossings.

It is worth pointing out from a physical point of view that, since the symmetry of the ground state eigenfunction changes from symmetric to antisymmetric for any $\beta>\beta_{0}$, the model exhibits a quantum phase transition. Furthermore, the effect produced by the singular $\delta^{\prime}$-perturbation is far stronger than the Zeldovich effect produced by the $\delta$-interaction due to the fact that the horizontal asymptotes of the curves are situated below the horizontal lines pertaining to the unperturbed symmetric eigenvalues (see [15-17] for a detailed description of the spectrum of the 1D harmonic oscillator perturbed by Dirac distributions). 


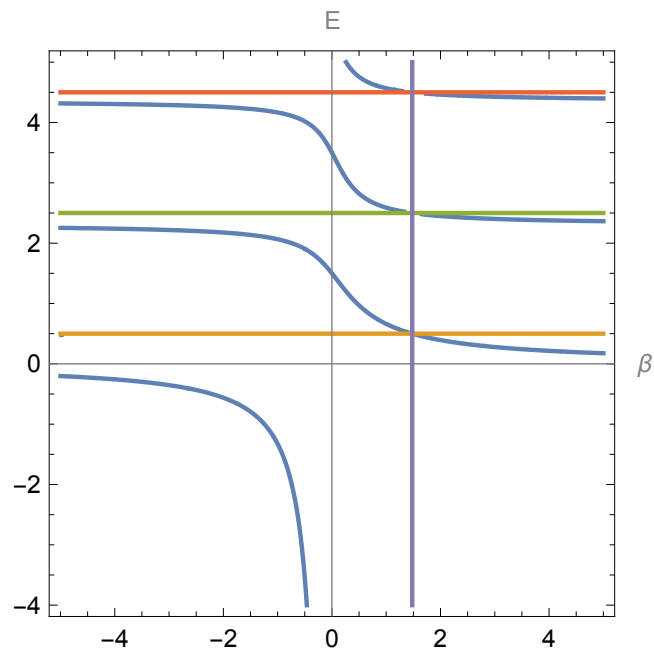

FIG. 1. The curves of the four lowest eigenenergies of $H_{\beta}$ as functions of the coupling parameter $\beta$, and the level crossings occurring at $\beta=\beta_{0}=\frac{\Gamma(1 / 4)}{2 \Gamma(3 / 4)} \approx 1.47934$. The horizontal lines are the even energy levels of the unperturbed harmonic oscillator: $E_{0}=1 / 2$ (yellow), $E_{2}=5 / 2$ (green), $E_{4}=9 / 2$ (red)

The same spectral pattern has recently been encountered in the discrete spectrum of the self-adjoint Hamiltonian of the conic oscillator $H_{\beta}^{c}$ acting like $H_{0}^{c}=\frac{1}{2}\left[-\frac{d^{2}}{d x^{2}}+|x|\right]$ on functions satisfying the condition $\psi\left(0^{+}\right)-\psi\left(0^{-}\right)=$ $-\beta \psi^{\prime}(0)$, whose resolvent is explicitly given by (see $[12,13]$ for the procedure requiring renormalization needed to define $\left.H_{\beta}^{c}\right)$ :

with:

$$
\left[H_{\beta}^{c}-E\right]^{-1}=\left[H_{0}-E\right]^{-1}+\frac{\left|\Psi^{c}(E)\right\rangle\left\langle\Psi^{c}(E)\right|}{\frac{1}{\beta}-\frac{\operatorname{Ai}(0) \mathrm{Ai}^{\prime}(-2 E)-\mathrm{Ai}^{\prime}(0) \operatorname{Ai}(-2 E)}{\operatorname{Ai}(0) \operatorname{Ai}(-2 E)}},
$$

$$
\Psi^{c}(x ; E)=\frac{1}{2^{1 / 2}} \sum_{n=1}^{\infty} \frac{\psi_{2 n}(x)}{\left(E_{2 n}-E\right)},
$$

where $\psi_{2 n}(x)$ is the $2 n$-th normalized eigenfunction (pertaining to an antisymmetric bound state) of $H_{0}^{c}$ and $E_{2 n}$ the corresponding eigenvalue, fully investigated in [18]. The function $\Psi^{c}(x ; E)$ is also a square summable function since the sequence $\left(E_{2 n}-E\right)^{-1}$ behaves like $n^{-2 / 3}$ as $n \rightarrow \infty$ and has a jump discontinuity at the origin satisfying the condition $\psi\left(0^{+}\right)-\psi\left(0^{-}\right)=-\beta \psi^{\prime}(0)$.

As a consequence, the bound state equation reads:

$$
\frac{1}{\beta}=\frac{\operatorname{Ai}(0) \operatorname{Ai}^{\prime}(-2 E)-\operatorname{Ai}^{\prime}(0) \operatorname{Ai}(-2 E)}{\operatorname{Ai}(0) \operatorname{Ai}(-2 E)},
$$

or equivalently:

$$
\beta=\frac{\operatorname{Ai}(0) \operatorname{Ai}(-2 E)}{\operatorname{Ai}(0) \operatorname{Ai}^{\prime}(-2 E)-\operatorname{Ai}^{\prime}(0) \operatorname{Ai}(-2 E)} .
$$

Given that $\mathrm{Ai}^{\prime}\left(-2 E_{2 n-1}\right)=0$ for any $n \geq 1$, it follows that, if the energy is exactly equal to any odd eigenvalue (pertaining to a symmetric bound state), the right hand side of (7) is equal to:

$$
\beta_{0}^{c}=-\frac{\operatorname{Ai}(0)}{\operatorname{Ai}^{\prime}(0)},
$$

which implies that such eigenvalues cause the denominator of the rank one operator on the right hand side of (4) for the special value of the coupling parameter $\beta=\beta_{0}^{c}=-\frac{\operatorname{Ai}(0)}{\operatorname{Ai}^{\prime}(0)} \approx 1.37172$ to vanish. This leads to their double degeneracy as eigenvalues of the Hamiltonian $H_{\beta_{0}^{c}}^{c}$ and the ensures level crossings at that particular value of the coupling. 
The blue curves in Fig. 2 depict $E_{0}(\beta), E_{2}(\beta)$ and $E_{4}(\beta)$, the three lowest lying energy levels pertaining to antisymmetric bound states generated by the singular perturbation as functions of the coupling parameter. The yellow horizontal line represents $E_{1}$ and the green one $E_{3}$, the energies of the two lowest symmetric bound states. Quite obviously, the spacing between two consecutive energy levels of $H_{0}^{c}$, the Hamiltonian of the unperturbed conic oscillator, is not constant in contrast with what happens for the harmonic oscillator. Nevertheless, the spectral features are qualitatively identical to those observed in the latter case: all the level crossings take place for the same value of the coupling parameter which is located to the right of the origin. Identically to what has already been seen in the case of the harmonic oscillator, the effect produced by this perturbation is far stronger than the one named after Zeldovich occurring for an attractive $\delta$-interaction.

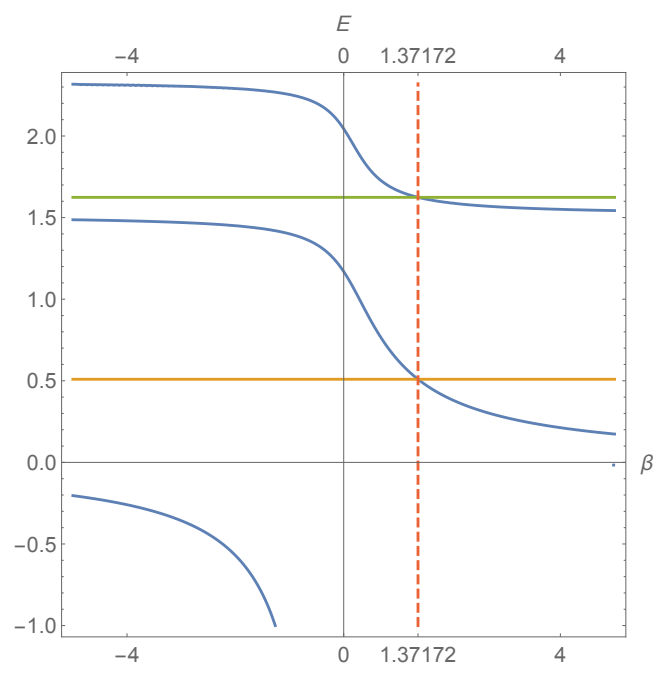

FIG. 2. The curves of the three lowest eigenenergies as functions of the coupling parameter $\beta$, and the level crossings occurring at $\beta=\beta_{0}^{c}=-\frac{\mathrm{Ai}(0)}{\operatorname{Ai}^{\prime}(0)} \approx 1.37172$. The horizontal lines are the energy levels of the unperturbed conic oscillator: $E_{1}$ (yellow) and $E_{3}$ (green)

\section{Level crossings in the discrete spectrum of the three-dimensional isotropic harmonic oscillator perturbed by a central $\delta$-interaction}

It is well known that the three-dimensional negative Laplacian perturbed by the Dirac distribution cannot be defined by means of the KLMN theorem since such a perturbation is no longer infinitesimally small in the sense of quadratic forms (see [1,2]). The same obviously holds in the presence of an harmonic confining potential.

The self-adjoint operator $H_{\beta}$, representing the Hamiltonian of he three-dimensional isotropic harmonic oscillator perturbed by a central $\delta$-interaction, defined either by means of the self-adjoint extension method or renormalization, has been investigated in depth in [7-10]. The entirely discrete spectrum of the operator, in particular the lowest lying eigenvalues, has also been studied in detail in those papers. The operator is rigorously defined by means of the explicit formula for its resolvent, namely:

$$
\left[H_{\beta}-E\right]^{-1}=\left[H_{0}-E\right]^{-1}+\frac{2|\Psi(E)\rangle\langle\Psi(E)|}{\frac{1}{\beta}-\frac{1}{\beta_{0}}+\frac{\Gamma(3 / 4-E / 2)}{\pi \Gamma(1 / 4-E / 2)}}
$$

with $\beta_{0}=\frac{\pi \Gamma(1 / 4)}{\Gamma(3 / 4)} \approx 9.29495$ and:

$$
\Psi(x, y, z ; E)=\sum_{n_{1}=0}^{\infty} \sum_{n_{2}=0}^{\infty} \sum_{n_{3}=0}^{\infty} \frac{\psi_{2 n_{1}}(0) \psi_{2 n_{2}}(0) \psi_{2 n_{3}}(0)}{2 n_{1}+2 n_{2}+2 n_{3}+\frac{3}{2}-E} \psi_{2 n_{1}}(x) \psi_{2 n_{2}}(y) \psi_{2 n_{3}}(z),
$$

whose norm, for any $E<0$, is bounded by:

$$
\left[\sum_{n=0}^{\infty} \frac{\psi_{2 n}^{2}(0)}{\left(2 n+\frac{1}{2}\right)^{2 / 3}}\right]^{3 / 2}<\infty
$$


since the sequence $\psi_{2 n}(0)$ behaves like $n^{-1 / 4}$ as $n \rightarrow \infty$.

As is well known, the eigenenergy $2 n_{1}+2 n_{2}+2 n_{3}+\frac{3}{2}$ in the discrete spectrum of the three-dimensional isotropic harmonic oscillator has degeneracy $\frac{1}{2}\left(2 n_{1}+2 n_{2}+2 n_{3}+1\right)\left(2 n_{1}+2 n_{2}+2 n_{3}+2\right)$. Out of all the eigenstates sharing this energy, only the one having radial symmetry will be affected by the 3D point perturbation and give rise to a new simple eigenvalue, thus lowering the original degeneracy of the level to $\frac{1}{2}\left(2 n_{1}+2 n_{2}+\right.$ $\left.2 n_{3}+1\right)\left(2 n_{1}+2 n_{2}+2 n_{3}+2\right)-1$. This new simple eigenvalue is determined as a function of the coupling parameter by the equation:

$$
\frac{1}{\beta_{0}}-\frac{1}{\beta}=\frac{\Gamma(3 / 4-E / 2)}{\pi \Gamma(1 / 4-E / 2)} .
$$

It is worth noting that, apart from the different constant multiplying the ratio of Gamma functions on the right hand side, the latter equation is of the same type of (3). However, the zeros of the entire function $\frac{1}{\pi \Gamma(1 / 4-E / 2)}$ are now given by $E=2 N+\frac{5}{2}$, the eigenenergies pertaining to the antisymmetric states, so that for the particular value $\beta=\beta_{0}$ the degeneracy of the next lower antisymmetric state increases by one. Hence, we get level crossings occurring for the unique value of the coupling parameter, namely $\beta_{0}=\frac{\pi \Gamma(1 / 4)}{\Gamma(3 / 4)} \approx 9.29495$. Therefore, the 3D point perturbation must be strongly attractive in order to exhibit the level crossing phenomenon.

In Fig. 3, the lowest level crossings between the new eigenvalues created by the point perturbation and the antisymmetric levels $E=\frac{5}{2}, \frac{9}{2}, \frac{13}{2}$, clearly unaffected by the singular perturbation, are depicted. Therefore, also in the three-dimensional case, the effect is stronger than the Zeldovich effect that would occur in the presence of any short range interaction.

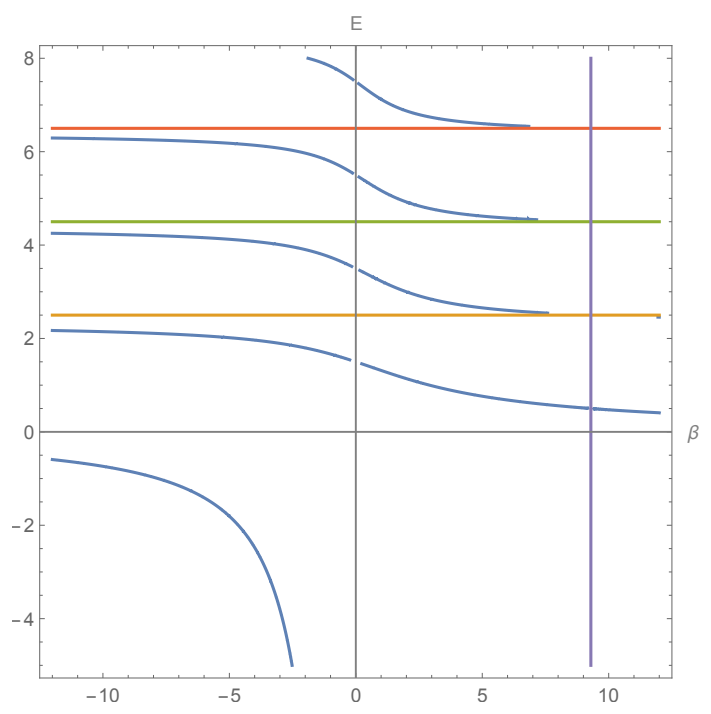

FIG. 3. The three lowest level crossings between the new eigenvalues created by the point perturbation and the antisymmetric levels $E=\frac{5}{2}, \frac{9}{2}, \frac{13}{2}$, clearly unaffected by the singular perturbation

\section{Level crossings in the discrete spectrum of the two-dimensional isotropic harmonic oscillator perturbed by a central $\delta$-interaction}

In perfect analogy with the three-dimensional case, the two-dimensional self-adjoint Hamiltonian $H_{\beta}$ representing the Hamiltonian of the two-dimensional isotropic harmonic oscillator perturbed by a central $\delta$-interaction, can be defined by means of the self-adjoint extension theory or by renormalization. Its discrete spectrum, in particular the lowest lying eigenvalues, has been investigated far less than the three-dimensional analog (see $[19,20]$ ).

As the intermediate steps would be identical to those for the three-dimensional case (see [7]), we omit them and write the limit as $N \rightarrow \infty$ in the norm topology of the resolvents, that is to say the counterpart of (4) in [7]: 


$$
\left(H_{\beta}-E\right)^{-1}=\left(H_{0}-E\right)^{-1}+\frac{|\Psi(E)\rangle\langle\Psi(E)|}{\frac{1}{\beta}-E \sum_{n_{1}=0}^{\infty} \sum_{n_{2}=0}^{\infty} \frac{\psi_{2 n_{1}}^{2}(0) \psi_{2 n_{2}}^{2}(0)}{\left(2 n_{1}+2 n_{2}+1\right)\left(2 n_{1}+2 n_{2}+1-E\right)}}
$$

with:

$$
\Psi(x, y ; E)=\sum_{n_{1}=0}^{\infty} \sum_{n_{2}=0}^{\infty} \frac{\psi_{2 n_{1}}(0) \psi_{2 n_{2}}(0)}{2 n_{1}+2 n_{2}+1-E} \psi_{2 n_{1}}(x) \psi_{2 n_{2}}(y),
$$

whose norm, for any $E<0$, is bounded by:

$$
\sum_{n=0}^{\infty} \frac{\psi_{2 n}^{2}(0)}{\left(2 n+\frac{1}{2}\right)}<\infty
$$

since the sequence $\psi_{2 n}(0)$ behaves like $n^{-1 / 4}$ as $n \rightarrow \infty$.

Given that the eigenvalues of the operator $H_{\beta}$ are exactly the poles of its resolvent, in order to achieve an accurate description of the spectrum (obviously being exclusively discrete) of the operator, we need only look for the solutions of the equation:

$$
\frac{1}{\beta}=E \sum_{n_{1}=0}^{\infty} \sum_{n_{2}=0}^{\infty} \frac{\psi_{2 n_{1}}^{2}(0) \psi_{2 n_{2}}^{2}(0)}{\left(2 n_{1}+2 n_{2}+1\right)\left(2 n_{1}+2 n_{2}+1-E\right)} .
$$

The striking difference between the latter equation and its three-dimensional counterpart is that the right hand side cannot be recast as a ratio of Gamma functions; this can be seen as follows. By exploiting the well-known integral relationship between the resolvent and the semigroup for any semibounded operator for any $E$ below the lowest point in the spectrum (see, e.g., [21] page 204), as well as the fact that the integral kernel of the semigroup of the two-dimensional harmonic oscillator is perfectly separable, we get that the right hand side of the above equation reads for any $E<1$ :

$$
\frac{1}{\beta}=\frac{1}{\pi} \int_{0}^{\infty} \frac{e^{t}\left(e^{E t}-1\right)}{e^{2 t}-1} d t=\frac{1}{\pi} \int_{0}^{\infty} \frac{e^{-t}\left(e^{E t}-1\right)}{1-e^{-2 t}} d t=\frac{1}{\pi} \int_{0}^{1} \frac{\xi^{-E}-1}{1-\xi^{2}} d \xi .
$$

It is not difficult to show that the last integral on the right hand side can be recast as a limit of an expression involving Gamma functions so that the bound state equation becomes:

$$
\alpha=\frac{1}{2 \pi} \lim _{\varepsilon \rightarrow 0_{+}} \Gamma(\varepsilon)\left[\frac{\Gamma(1 / 2-E / 2)}{\Gamma(1 / 2-E / 2+\varepsilon)}-\frac{\Gamma(1 / 2)}{\Gamma(1 / 2+\varepsilon)}\right] .
$$

By exploiting the well-known identity:

and the limits:

$$
\Gamma(\varepsilon) \Gamma(1-\varepsilon)=\frac{\pi}{\sin (\pi \varepsilon)},
$$

$$
\lim _{\varepsilon \rightarrow 0_{+}} \frac{\sin (\pi \varepsilon)}{\varepsilon}=\pi, \quad \quad \lim _{\varepsilon \rightarrow 0_{+}} \Gamma(1-\varepsilon)=1,
$$

equation (18) can be transformed into:

$$
\alpha=\frac{1}{2 \pi} \lim _{\epsilon \rightarrow 0_{+}} \frac{1}{\epsilon}\left[\frac{\Gamma(1 / 2-E / 2)}{\Gamma(1 / 2-E / 2+\epsilon)}-\frac{\Gamma(1 / 2)}{\Gamma(1 / 2+\epsilon)}\right] .
$$

The limit on the right hand side exists, which leads to an expression involving the difference of two different values of the logarithmic derivative of the Gamma function, namely:

By noting that:

$$
\frac{1}{2 \pi}\left[\frac{\Gamma^{\prime}(1 / 2)}{\Gamma(1 / 2)}-\frac{\Gamma^{\prime}(1 / 2-E / 2)}{\Gamma(1 / 2-E / 2)}\right]=\frac{1}{2 \pi}\left[(\ln \Gamma)^{\prime}(1 / 2)-(\ln \Gamma)^{\prime}(1 / 2-E / 2)\right] .
$$

$$
(\ln \Gamma)^{\prime}(x)=F(x-1)=-\gamma-\sum_{n=1}^{\infty}\left(\frac{1}{n+x-1}-\frac{1}{n}\right),
$$

where $\gamma$ denotes the Euler-Mascheroni constant and $F(x-1)$ is the Digamma function, the latter expression can be recast as:

$$
\frac{1}{2 \pi}[F(-1 / 2)-F(-1 / 2-E / 2)]=\frac{E}{4 \pi} \sum_{n=1}^{\infty} \frac{1}{\left(n-\frac{1}{2}\right)\left(n-\frac{1+E}{2}\right)} .
$$


Hence, the equation determining the perturbed eigenvalues of our Hamiltonian can be written either in terms of the difference of two different values of the Digamma function or in terms of a simple series (reducing to a telescoping one when $E$ is any integer with the exclusion of all odd positive ones):

$$
\frac{1}{\beta}=\frac{1}{2 \pi}[F(-1 / 2)-F(-1 / 2-E / 2)]=\frac{E}{4 \pi} \sum_{n=0}^{\infty} \frac{1}{\left(n+\frac{1}{2}\right)\left(n+\frac{1-E}{2}\right)} .
$$

In particular, for any $E$ given by a positive even number, the total energy of an antisymmetric state, the series on the right hand side becomes a simple telescoping series so that it is rather straightforward to find the location of the points where level crossings occur. For the lowest antisymmetric level with $E=2$ we find $\beta_{1}=-\pi$, whereas for the upper one $E=4$ we have $\beta_{2}=-\frac{3}{4} \pi$.

Figure 4 depicts the two lowest level crossings between the new eigenvalues of the perturbed Hamiltonian as functions of the strength of the point interaction and the two lowest antisymmetric levels of the 2D harmonic oscillator clearly belonging to the spectrum of perturbed Hamiltonian. As anticipated in the introduction, the first striking difference between Fig. 4 and Fig. 3, its three-dimensional counterpart, is that in the plot of the twodimensional model the level crossings take place to the left of the origin, which implies that in two dimensions the point perturbation need only be weakly repulsive to produce the level crossing. Furthermore, each level crossing occurs at a different value of the coupling parameter.



FIG. 4. Plot of the two lowest level crossings between the eigenvalues created by the point perturbation and the two lowest antisymmetric levels of the harmonic oscillator which also belong to the spectrum of the perturbed Hamiltonian

\section{Final remarks}

The spectral phenomenon known as "level crossing of eigenvalues" has been investigated by considering the entirely discrete spectrum of four different energy operators: the Hamiltonian of the one-dimensional harmonic oscillator perturbed by a nonlocal $\delta^{\prime}$-interaction, the Hamiltonian of the one-dimensional conic oscillator perturbed by the same singular interaction, the Hamiltonian of the two-dimensional harmonic oscillator perturbed by a $\delta$ interaction and its three-dimensional analog. All the point perturbations considered are highly singular so that the KLMN theorem used to define the $\delta$-perturbation in one dimension may not be invoked.

Although the level crossings in the one-dimensional models share with the three-dimensional model the property of occurring for a unique positive value of the coupling parameter (representing an attractive interaction), their nature is fundamentally different since in one dimension the eigenvalues pertaining to the antisymmetric bound states created by the singular perturbation cross the unperturbed even energy levels, exactly the opposite of what happens in three dimensions.

The two-dimensional model exhibits entirely different features; the crossings take place for different negative values of the coupling parameter so that the singular interaction need only be weakly repulsive to produce the crossings. From a mathematical point of view, the difference is due to the absence of a ratio of Gamma functions in the bound state equation and the appearance of a difference of Digamma functions as a consequence of the 
logarithmic divergences of two-dimensional quantum mechanics. Further work to achieve a deeper understanding of the two-dimensional model is ongoing.

\section{Acknowledgements}

S. Fassari wishes to acknowledge Prof. I. Popov's kind invitation to present the main points of this article in one of the sessions of the conference "Mathematical Challenge of Quantum Transport in Nanosystems, Pierre Duclos Workshop", held at ITMO University, Saint Petersburg, Russia (September 26-27, 2017). Stimulating discussions with Prof. M. J. Lopez and Prof. J. Negro are kindly acknowledged. Partial financial support is acknowledged to the Spanish Junta de Castilla y León and FEDER (Project VA057U16) and MINECO (Project MTM2014-57129-C2-1-P). S. Fassari and M. L. Glasser also wish to thank the entire staff at Departamento de Física Teórica, Atómica y Óptica, Universidad de Valladolid, for their warm hospitality throughout their stays.

\section{References}

[1] Albeverio S., Gesztesy F., Høegh-Krohn R., Holden H. Solvable Models in Quantum Mechanics (AMS Chelsea Series), 2nd edition, American Mathematical Society, Providence, RI, 2004.

[2] Albeverio S., Kurasov P. Singular Perturbations of Differential Operators. Publishing House, Cambridge UK, 2000.

[3] Albeverio S., Dabrowski L., Kurasov P. Symmetries of Schrödinger operators with point interactions. Lett. Math. Phys., 1998 , 45, P. 33-47.

[4] Exner P., Neidhardt H., Zagrebnov V.A. Potential approximations to $\delta^{\prime}$ : an inverse Klauder phenomenon with norm-resolvent convergence. Commun. Math. Phys., 2001, 224, P. 593-612.

[5] Fassari S., Rinaldi F. On the spectrum of the Schrödinger Hamiltonian with a particular configuration of three point interactions. Rep. Math. Phys., 2009, 64, P. 367-393.

[6] Albeverio S., Fassari S., Rinaldi F. The Hamiltonian of the harmonic oscillator with an attractive $\delta^{\prime}$-interaction centred at the origin as approximated by the one with a triple of attractive $\delta$-interactions. Journal of Physics A: Mathematical and Theoretical, $2016,49,025302$.

[7] Fassari S., Inglese G. Spectroscopy of a three-dimensional isotropic harmonic oscillator with a delta-type perturbation. Helv. Phys. Acta, 1996, 69, P. 130-140.

[8] Brüning J., Geyler V., Lobanov I. Spectral properties of a short-range impurity in a quantum dot. J. Math. Phys., 2004, 45, P. 1267-1290.

[9] Albeverio S., Fassari S., Rinaldi F. Spectral properties of a symmetric three-dimensional quantum dot with a pair of identical attractive $\delta$-impurities symmetrically situated around the origin II. Nanosystems: Physics, Chemistry, Mathematics, 2016,7 (5), P. 803-815.

[10] Albeverio S., Fassari S., Rinaldi F. Spectral properties of a symmetric three-dimensional quantum dot with a pair of identical attractive $\delta$-impurities symmetrically situated around the origin. Nanosystems: Physics, Chemistry, Mathematics, 2016,7 (2), P. $268-289$.

[11] Albeverio S., Fassari S., Rinaldi F. A remarkable spectral feature of the Schrödinger Hamiltonian of the harmonic oscillator perturbed by an attractive $\delta^{\prime}$-interaction centred at the origin: double degeneracy and level crossing. Journal of Physics A: Mathematical and Theoretical, 2013, 46, 385305.

[12] Fassari S., Gadella M., Glasser M.L., Nieto L.M. On the spectrum of the Schrödinger Hamiltonian of the one-dimensional conic oscillator perturbed by a point interaction. ArXiv preprint 1706.04916, 2017.

[13] Fassari S., Gadella M., Glasser M.L., Nieto L.M. Spectroscopy of a one-dimensional V-shaped quantum well with a point impurity. Annals of Physics, 2018, 389, P. 48-62.

[14] Cheon T., Shigehara T. Fermion-Boson duality of one-dimensional quantum particles with generalized contact interactions. Phys. Rev. Lett., 1999, 82, 2536.

[15] Fassari S., Inglese G. On the spectrum of the harmonic oscillator with a delta-type perturbation. Helv. Phys. Acta, 1994, 67, P. 650-659.

[16] Fassari S., Inglese G. On the spectrum of the harmonic oscillator with a delta-type perturbation. II. Helv. Phys. Acta, 1997, 70, P. 858-865.

[17] Fassari S., Rinaldi F. On the spectrum of the Schrödinger Hamiltonian of the one-dimensional harmonic oscillator perturbed by two identical attractive point interactions. Rep. Math. Phys., 2012, 69, P. 353-370.

[18] Lörinczi J., Ma-lecki J. Spectral properties of the massless relativistic harmonic oscillator. J. Diff. Eq., 2012, 253, P. 2846-2871.

[19] Tusek M. Analysis of Two-Dimensional Quantum Models with Singular Potentials, Diploma Thesis, Czech Technical University, Prague, 2006.

[20] Tunali S. Point Interactions in Quantum Mechanics, MS Thesis, Izmir Institute of Technology, Izmir, 2014.

[21] Reed M., Simon B. Methods in Modern Mathematical Physics IV - Analysis of Operators, Academic Press, NY, 1978. 